\section{Divers advisers}

\section{Stuart Sutherland}

New Ideas in Psychology: An

International Journal of Innovative

Theory in Psychology.

Editors Pierre Moessinger, John

Broughton and Richard Kitchener.

Pergamon. 3/yr. £46, \$80.

PSYCHOLOGY is becoming a spectator sport. From the terraces a motley crowd of philosophers, linguists, sociologists, anthropologists and artificial intelligencers heckle, cheer and boo. But above all they proffer advice.

The first four issues of New Ideas in Psychology (NIP) contain a good many papers of this kind. Some authors advise psychologists to revert to teleological explanations, others urge them to seek salvation in artificial intelligence. Yet others recommend speculative theories provided they are testable, while perhaps the most original piece of advice is contained in a paper which advocates the founding of a science of happiness, to be known as eudology.

Almost all the papers are followed by commentaries, a format that increases the interest of NIP, which is nothing if not eclectic. There are papers on such disparate topics as chimpanzee language, brief psychoanalytic therapy, personal construct theory and the existence of paranormal phenomena, and there is a solitary experimental report that suggests laughing gas operates through the orbital frontal cortex.

Although there is room for a psychological journal of speculation, one wonders whether the fields covered by NIP are not too diverse. Moreover, the quality of the contributions is a little uneven and one commentator's remarks on an article apply to several. He writes,

Like most programmatic manifestoes it serves the socio-psychological purpose of alerting likeminded scholars to the existence of a community of concern, but it fails to show signs of that rigor of analysis and systematic coverage of the subject matter that the author claims must distinguish a science.

The journal should provide an outlet for articles of this kind and will therefore doubtless serve an important "sociopsychological purpose'".

Stuart Sutherland is Director of the Centre for Research on Perception and Cognition, University of Sussex.

\section{Split psychology}

\section{Geoffrey Hall}

Journal of Comparative Psychology.

Editor Jerry Hirsch.

American Psychological Association.

4/yr. \$36 (US), $\$ 39$ (elsewhere).

Behavioral Neuroscience.

Editor Richard F. Thompson.

American Psychological Association.

6/yr. \$100 (US), \$103 (elsewhere).

THE influence of the American Psychological Association is such that when it decides to reorganize its journals the international psychological community has to take notice. If a shift in policy does not reflect a change of emphasis in work in psychology, then it seems probable that one will result.

These two "new" journals are the fission products of the demise of the old Journal of Comparative and Physiological Psychology (JCPP) which existed from 1947 to 1982 as the continuation of an earlier (1921-1946) Journal of Comparative Psychology (JCP). The original JCP attempted to cover all aspects of the behaviour of non-human animals; its change of title recognized that physiological studies (of brain lesions and, latterly, of the effects of drugs) had come to predominate. When, in 1975, the other main line of work covered by JCPP (laboratory studies of conditioning and learning) was hived off as a special section of the Journal of Experimental Psychology, a reconsideration seemed appropriate. What we have now been given in Behavioral Neuroscience is a journal devoted to "the broad field of biological bases of behavior" whereas the new version of JCP is concerned with "behavioral patterns ... . as they relate to evolution, development... and functional significance".

Contributors to a recent symposium on "the place of physiological psychology" saw in this split evidence of an identity crisis in their discipline. But in fact Behavioral Neuroscience with its sophisticated behavioural analyses of the effects of lesions and of drugs looks like a direct continuation of JCPP. The work reported is first-rate and this journal will retain its predecessor's reputation as being foremost in its field. JCP is something rather new but seems set to establish itself as a direct competitor to the respected ethological journal Animal Behaviour. Given the recent upsurge of interest in North America in what is sometimes called the "ecological" approach to animal behaviour, there is much good work to report and plenty of room for both journals.

These various divisions seem to follow natural fracture lines and are a sensible reaction to the growth of the field. Nonetheless, one looks back on the original JCP with a tinge of regret - ethologists have things of interest to say to learning theorists and both should communicate with physiological psychologists; it would be a pity if any new journal structure were to make such interactions less likely.

Geoffrey Hall is Senior Lecturer in Psychology at the University of York.

\section{Nervous beginnings}

\author{
A.G.E. Pearse
}

Neurochemical Pathology.

Editor-in-chief Lloyd A. Horrocks.

Humana. 4/yr. $\$ 100$.

CHemical pathology, a term once popular, now has a decidedly antediluvian flavour, following absorption of the subject into clinical biochemistry. So what is neurochemical pathology except neuropathology in modern guise?

It comes as no surprise to learn that that is apparently just what the editors of this journal intended. According to their stated terms of reference, the journal is to "serve as the key forum for studies in the rapidly expanding area of biochemical neuropathology" and it "will thus be predominantly devoted to biochemical studies on all those tissues or body fluids that may help to elucidate the etiology or pathogenesis of neurological disorders".

Neurochemical Pathology is sponsored by the International Society for Neurochemistry and the World Federation of Neurology Research Groups on Neurochemistry and Cerebrospinal Fluid. I recognize, on the editorial board, many names derived from these two sources but I doubt if all were consulted in the matter of the creation of the new journal.

It may be pertinent to enquire, in so far as the first volume is concerned, just how far the editors have succeeded in their reasonably justifiable aims. There are 21 papers in the four numbers comprising the volume, and of these no less than 14 come into the category of experimental neuropathology. There are three purely neurochemical papers, each of which contains only a hint of future pathological importance, and three further papers describing chemical aspects of known, little known or unknown syndromes. The one remaining paper is an exercise in clinical neurochemistry.

With so great a preponderance of reports on experimental work - and despite the fact that much of it has some relevance, at least, to deficiencies or overdosages potentially occurring in human beings - one wonders whether the word "experimental" should not have been included in the journal's title. But it is clear enough that the editors did not intend their first year's coverage to present to the reader this predominantly experimental front, nor the virtual exclusion of several of their specifically cited areas of interest.

I conclude that several more years (more volumes) will require surveyance before it is possible to reach a positive conclusion that this journal is a useful one. Sad to say, with its present format and content it fails to justify its existence at all.

Anthony Pearse is Professor Emeritus of Histochemistry in the University of London. 